Published in final edited form as:

Nat Commun. ; 5: 4339. doi:10.1038/ncomms5339.

\title{
The highly conserved domain of unknown function 1792 has a distinct glycosyltransferase fold
}

\author{
Hua Zhang ${ }^{1}$, Fan Zhu ${ }^{1}$, Tiandi Yang ${ }^{2}$, Lei Ding ${ }^{3}$, Meixian Zhou ${ }^{1, \#}$, Jingzhi Li ${ }^{4}$, Stuart M \\ Haslam $^{2}$, Anne Dell ${ }^{2}$, Heidi Erlandsen ${ }^{5,6}$, and Hui $\mathrm{Wu}^{1,{ }^{*}}$ \\ ${ }^{1}$ Departments of Pediatric Dentistry, Microbiology, University of Alabama at Birmingham, Schools \\ of Dentistry and Medicine, Birmingham, AL35294 \\ 2Department of Life Sciences, Imperial College London, London SW7 2AZ, UK \\ ${ }^{3}$ Department of Biochemistry and Molecular Genetics, University of Alabama at Birmingham, \\ Schools of Dentistry and Medicine, Birmingham, AL35294 \\ ${ }^{4}$ Department of Cell Biology, University of Alabama at Birmingham, Schools of Dentistry and \\ Medicine, Birmingham, AL35294 \\ ${ }^{5}$ Department of Periodontology, University of Alabama at Birmingham, Schools of Dentistry and \\ Medicine, Birmingham, AL35294 \\ ${ }^{6}$ Institute of Oral Health Research, University of Alabama at Birmingham, Schools of Dentistry \\ and Medicine, Birmingham, AL35294
}

\begin{abstract}
More than 33,000 glycosyltransferases have been identified. Structural studies, however, have only revealed two distinct glycosyltransferase (GT) folds, GT-A and GT-B. Here we report a 1.34 $\AA$ resolution X-ray crystallographic structure of a previously uncharacterized "domain of unknown function" 1792 (DUF1792) and show that the domain adopts a new fold and is required for glycosylation of a family of serine-rich repeat streptococcal adhesins. Biochemical studies reveal that the domain is a glucosyltransferase, and it catalyzes the transfer of glucose to the branch point of the hexasaccharide O-linked to the serine-rich repeat of the bacterial adhesin, Fap1 of Streptococcus parasanguinis. DUF1792 homologs from both Gram-positive and Gram-negative bacteria also exhibit the activity. Thus DUF1792 represents a new family of glycosyltransferases,
\end{abstract}

Users may view, print, copy, and download text and data-mine the content in such documents, for the purposes of academic research, subject always to the full Conditions of use:http://www.nature.com/authors/editorial_policies/license.html\#terms

*Correspondence should be addressed to Hui Wu. hwu@uab.edu.

\#Current Address: Key Laboratory of Marine Biogenetic Resources, Third Institute of Oceanography, State Oceanic Administration, Xiamen 361005, China

Author contributions

Hua Zhang, Hui Wu, Anne Dell and Stuart M Haslam designed the study; Hua Zhang and Fan Zhu performed the structure/function experiments; Hua Zhang, Fan Zhu and Meixian Zhou and Hui Wu analyzed the structure/function experimental data; Lei Ding and Jingzhi Li collected X-ray data and performed modelling; Hua Zhang and Heidi Erlandsen analyzed the structural results. Tiandi Yang performed glycan structure analysis; Tiandi Yang, Stuart M Haslam and Anne Dell analyzed MS data; Hua Zhang, Hui Wu, Anne Dell, Stuart M Haslam and Tiandi Yang wrote the paper.

Competing financial interests: The authors declare no competing financial interests.

Accession codes: Coordinates and structure factors for DUF1792-Mn, DUF1792-native and DUF1792 (Se-Met) crystal structures have been deposited in the Protein Data Bank with the succession numbers 4PHR, 4PFX and 4PHS respectively. 
so we designate it as a GT-D glycosyltransferase fold. As the domain is highly conserved in bacteria and not found in eukaryotes, it can be explored as a new antibacterial target.

\section{Keywords}

streptococcal adhesin; glycosyltransferase; DUF1792

\section{INTRODUCTION}

Protein glycosylation, catalyzed by glycosyltransferases, is an important protein modification found in both prokaryotes ${ }^{1}$ and eukaryotes ${ }^{2}$ where it plays crucial roles in cellcell recognition, adhesion and intracellular sorting $3,4,5$. Since the classification system for glycosyltransferases based on amino acid sequence similarity was proposed by Campbell et al. in $1997^{6}$, the number of glycosyltransferases has grown enormously to over 33,000, organized into over 100 subfamilies $6,7,8$.

In contrast, numerous structural studies have revealed that the structural folds displayed by this large number of glycosyltransferases are limited and only two distinct structural folds, GT-A and GT-B have been rigorously characterized ${ }^{9,10}$. GT-A displays a single Rossmann fold (topology $\beta / \alpha / \beta / \alpha / \beta$ ) and a conserved 'DXD' metal-binding motif ${ }^{11,12}$. In contrast, GTB possesses twin Rossmann folds that face each other and are linked flexibly by the active site within the resulting cleft ${ }^{13}, 14$. In contrast this family does not require metal ions for its activity. There is another previously named glycosyltransferase fold, the GT-C fold. Recent structural studies of two predicted GT-C types of enzymes (oligosaccharyltransferase STT $3^{15}$ and peptidoglycan synthesizing glycosyltransferase PBP2 ${ }^{16,17}$ ) suggest that they actually adopt different protein folds. Thus, whether GT-C represents a distinct glycosyltransferase fold remains controversial.

Serine-rich repeat glycoproteins (SRRPs) are a growing family of bacterial adhesins and they play important roles in bacterial fitness and virulence ${ }^{18,19,20}$. Fimbriae-associated protein (Fap1) was the first SRRP identified ${ }^{21}$. It is heavily O-glycosylated by Glc-GlcNAclinked oligosaccharides containing up to four additional sugars ${ }^{22}$. Fap1 modulates bacterial biofilm formation in the oral bacterium Streptococcus parasanguinis ${ }^{23}$. Fap1-like SRRPs have since been identified from other streptococci ${ }^{24,25,26}$, staphylococci ${ }^{27,28,29}$ and other Gram-positive bacteria ${ }^{30}$. Biogenesis of Fap1 in S. parasanguinis is controlled by a gene cluster adjacent to this SRRP structural gene ${ }^{22}$. Analogous gene clusters are highly conserved in streptococci and staphylococci ${ }^{30}$. Glycosylation and secretion of Fap1 is mediated by eleven genes. A gene cluster coding for four putative glycosyltransferases, Gly, Gtf3, GalT1, and GalT2, is located upstream of fap 1, and another gene cluster producing accessory secretion components, SecY2, SecA2, Gap1, Gap2 and Gap3, and two putative glycosyltransferases (Gtf1 and Gtf2) is located downstream of fap $1^{31,32}$. Gtf1 and Gtf2 form a protein complex that catalyzes the first step of glycosylation by transferring GlcNAc residues to the Fap1 polypeptide ${ }^{31,33,34}$, while Gtf3 catalyzes the second step of glycosylation by transferring Glc residues to the GlcNAc-modified Fap $1^{35,36}$. However, it is not yet known which enzymes mediate the subsequent glycosylation steps. 
GalT1 in the fapl locus was annotated as a glycosyltransferase since the C-terminus of GalT1 is predicted to have a classic GT-A fold and shares significant homology with galactosyltransferases. A domain of unknown function is found at the N-terminus of GalT1, which belongs to an uncharacterized DUF1792 superfamily (c107392: DUF1792 Superfamily, commonly_found at the C-terminus of proteins that also contain the glycosyltransferase domain at the N-terminus). DUF1792 is highly conserved in numerous glycosyltransferases that have the same organization as exhibited in GalT1, and the DUF1792 domain module also exists by itself in streptococci, lactobacilli ${ }^{37}$ and even Gramnegative bacteria ${ }^{38}$. Sequence analysis and structural prediction reveal that DUF1792 does not share any homology with known glycosyltransferases, suggesting that it represents a new domain that may possess a unique activity.

In this study, we determine the glycan sequence on Fap1 and demonstrate that DUF1792 is a novel glucosyltransferase which catalyzes the third step of Fap1 glycosylation. Moreover, a $1.34 \AA$ resolution X-ray crystal structure of DUF1792 has revealed that DUF1792 is structurally distinct from all known GT folds of glycosyltransferases and contains a new metal binding site. The glycosyltransferase activity of DUF1792 appears to be highly conserved in pathogenic streptococci and fusobacteria. We conclude that DUF1792 represents a highly conserved glycosyltransferase superfamily with a novel GT fold and we designate this new glycosyltransferase fold as a GT-D type.

\section{RESULTS}

\section{Characterization of the O-glycans on Fap1}

We employed a variety of mass spectrometric glycomic strategies to characterize Fap1 glycosylation. Because it was difficult to isolate native Fap1 in sufficient quantities for indepth structure analysis, we first characterized the glycosylation of recombinant Fap1 which we obtained by co-expression of recombinant Fap1 ( $\mathrm{rFap} 1)^{35}$ with all the glycosyltransferases identified from the fapl locus. rFap1 was purified and subjected to beta-elimination to release the O-linked glycans for MS analysis. MALDI-TOF mass fingerprinting (Fig. 1a and b) of the beta-eliminated permethylated glycans showed a mixture of glycans ranging in size from a monosaccharide (hexose) up to a hexasaccharide comprised of one deoxyhexose, two HexNAcs and three hexoses. The latter is consistent with a previously reported monosaccharide composition for the native Fap1 glycan ${ }^{22}$. The smaller glycans correspond to biosynthetic precursors. Each peak from the glycan fingerprint was further analyzed by MALDI-TOF/TOF to generate glycan sequences. The MS/MS spectrum of the hexasaccharide peak at $m / z 1361.6$ is shown in Fig. 1(c). The data are fully consistent with the branched structure shown in the cartoon annotation on this figure. The identities of the sugars and their linkages were determined by additional GC-EIMS experiments. Sugar linkage analysis of partially methylated alditol acetates (Supplementary Table 1) determined rhamnose and glucose as non-reducing sugars in the hexasaccharide, and identified the reducing sugar as 6-linked GlcNAc. Other linkages observed were 3-linked GlcNAc, and 3- and 2,6-linked Glc, the latter being consistent with the branched sequence shown in Fig. 1(c). 
Collectively, the glycomics data show that the largest Fap1 glycan has the sequence Rha1-3Glc1-(Glc1-3GlcNAc1-)2,6Glc1-6GlcNAc. Moreover, the absence of a disaccharide intermediate in the glycomic fingerprints (Fig. 1a and b) suggests that there is a rapid incorporation of the second glucose in the biosynthetic pathway leading to the hexasaccharide. Also, since the same glycan fingerprint was observed in the native Fap1 purified from S. parasanguinis (Supplementary Fig. 1), we conclude that the latter shares the O-glycan sequences identified in our in-depth studies of recombinant samples.

\section{DUF1792 is required for the third step of Fap1 glycosylation}

While we have determined the first two steps of Fap1 glycosylation 34,36 the remaining glycosylation steps are unknown. In a search for proteins responsible for the subsequent steps of Fap1 glycosylation we identified dGT1 (previously named GalT1 because of its annotated function; we rename it as dGT1 as it has two functional domains). dGT1 is predicted to be a glycosyltransferase since it possesses a putative GT-A type glycosyltransferase domain at the $\mathrm{C}$-terminus. Interestingly, dGT1 also contains a distinct domain of unknown function DUF1792 at the N-terminus (Fig. 2a). In vitro glycosylation assays revealed that full-length dGT1 has a glucosyltransferase activity, transferring glucose residues to Glc-GlcNAc modified Fap1 (Fig. 2b), suggesting dGT1 is involved in the third step of Fap1 glycosylation. To dissect the individual dGT1 domain(s) involved, we expressed both the N-terminal DUF1792 domain (amino acids 1-272) and the C-terminal domain (amino acids 273-582), and determined their activity. Unexpectedly, the N-terminal DUF1792 domain, but not the predicted C-terminal glycosyltransferase GT-A domain is responsible for the in vitro glucosyltransferase activity (Fig. 2b). Moreover, the glucosyltransferase activity of DUF1792 is dependent on the presence of metal ions (Fig. 2c). $\mathrm{Mn}^{2+}$ maximized the activity. However DUF1792 does not have the classic metal binding motif, DXD, found in GT-A family of glycosyltransferases, suggesting that DUF1792 represents a new type of glycosyltransferase.

To further define the function of DUF1792, we examined the ability of DUF1792 to catalyze the third step of Fap1 glycosylation using a well-established E. coli glycosylation system ${ }^{35}$. Since we have demonstrated that Gtf1/2 and Gtf3 catalyze the first two steps of Fap1 glycosylation respectively, we co-expressed either DUF1792 or the full-length dGT1 with Gtf1/2, 3 and recombinant Fap1 (rFap1) ${ }^{35}$ to determine whether dGT1 or DUF1792 further glycosylates the Gtf1/2,3 modified rFap1. Indeed, dGT1 retarded the migration of the Gtf1/2,3 modified rFap1 (Fig. 3a, lane3 versus 2), suggesting additional modification by dGT1. Interestingly, the migration of the modified rFap1 was further retarded when coexpressed with the DUF1792 domain itself (Fig. 3a, lane 4). This is also true for the in vitro glycosyltransferase activity (Fig. 2b). The activity of DUF1792 is consistently higher than that from the full-length dGT1, suggesting the dGT1 C-terminus may have an additional unknown glycosyltransferase activity that coordinates with the function of DUF1792 in vitro. To further determine the relative contribution of DUF1792 and C-terminal dGT1 to Fap1 glycosylation in the native host S. parasanguinis, the dGT1 mutant of S. parasanguinis was complemented by either DUF1792 or C-terminal dGT1, and then examined by Fap1specific antibody mAbE42. The DUF1792 alone significantly retarded the migration of Fap1 indicative of glycosylation (Fig. 3b, lane 5) in comparison with the dGT1 mutant (Fig. 3b, 
lane 3) albeit it did not restore the migration as the full-length dGT1 (Fig. 3b, lane 4). By contrast, the C-terminal dGT1 failed to restore the migration, suggesting that the DUF1792 domain is more important than the C-terminal domain in vivo in S. parasanguinis, and that both domains are required for biogenesis of mature Fap1. The detailed function of the Cterminal domain and how it contributes to the Fap1 glycosylation, is under active investigation. DUF1792 is highly conserved in streptococci and several Gram-negative bacteria (Fig. 3c and Supplementary Fig. 2). It is also present in archea (Supplementary Fig. 2). To assess the functional conservation of DUF1792, we selected DUF1792 homologs from other streptococci and a Gram-negative bacterium, Fusobacterium nucleatum to evaluate whether they can further modify the Fap1 glycosylated by Gtf1/2, 3. All DUF1792 homologs (Fig. 3a, lanes 5-9 versus 2) retarded the migration of the Gtf123 modified Fap1, suggesting additional sugar residues were transferred to the Gtf123-modified Fap1.

To further confirm DUF1792 is capable of transferring Glc to the Glc-GlcNAc modified Fap1 revealed by in vitro glycosylation assays (Fig. 2b and c), we performed glycan profiling analysis. In the presence of DUF1792, the glycan mass of this recombinant Fap1 increased by a hexose increment (compare Fig. 4a and Fig. 4b), indicative of addition of Glc to the Glc-GlcNAc modified Fap1. Since sugar and linkage analyses (see above) established that the only hexose contained in rFap1 glycan is glucose, it is reasonable to deduce that DUF1792 has a glucosyltransferase activity. Moreover, by exploiting a glycomics strategy incorporating MS fingerprinting of peracetylated derivatives before and after chromium trioxide oxidation ${ }^{41}$, we showed that DUF1792 attaches the glucose in a beta anomeric linkage. Under the oxidation conditions employed, alpha linked peracetylated sugars are resistant to oxidation, while beta linked sugars are ring opened and oxidized, resulting in a mass shift of $14 \mathrm{Da}$ for each beta-linked sugar. The MALDI-TOF spectra of the peracetylated glycans synthesized by Gtf1/2/3 and DUF1792 before and after oxidation are shown in Fig. $4 \mathrm{c}$ and $\mathrm{d}$ respectively. The molecular ion of the oxidized glycan is shifted by $28 \mathrm{Da}$, which is attributable to two sugars being oxidized, indicating that both of the glucoses are beta-linked. Collectively the above data demonstrate the functional conservation of DUF1792 as a beta-glucosyltransferase.

\section{Overall structure of DUF1792}

To further characterize this highly conserved new family of glycosyltransferases, we solved the X-ray crystal structure of DUF1792 from S. parasanguinis. The structure of DUF1792 was built by selenomethionyl substituted protein X-ray data utilizing the MAD method (Table 1). Both native protein (Native-DUF1792) and the native protein in complex with Mn (DUF1792-Mn) crystallize in a space group of $\mathrm{C} 2$ and exist as a monomer. In the native structure, a UDP molecule and one acetate ion were found (Supplementary Fig. 3). In the DUF1792-Mn structure, a UDP, a manganese and two acetate ions were present (Fig. 5a and c).

The structure of DUF1792 consists of 277 residues organized into seven $\beta$-strands in the center, tightly surrounded by twelve $\alpha$-helices, which appear as a sandwich (Fig. 5a). Seven $\beta$-strands: $\beta 1$ (25-29), $\beta 2$ (67-70), $\beta 3$ (142-148), $\beta 4$ (165-171), $\beta 5$ (195-199), $\beta 6$ (217-220) and $\beta 7$ (269-271), form a parallel $\beta$-sheet in the topological order $\beta 2-\beta 1-\beta 6-\beta 5-\beta 3-\beta 4-\beta 7$. 
The sheet is flanked by eight helices a1 (1-6), a 5 (78-80), a6 (83-103), a2 (12-22), a7 (112-115), a8 (126-138), a 11 (224-233) and a12 (261-266) on one side, and four helices a3 (31-38), a4 (49-60), a9 (176-191) and a10 (202-214), on the other side.

The DUF1792 structure is composed of three regions: the $\mathrm{N}$-terminal region formed from helices $\alpha 1, \alpha 2, \alpha 3, \alpha 4, \alpha 5$ and two strands $\beta 1$ and $\beta 2 ; \alpha_{2} \beta_{1} a_{3}$ and $\alpha_{4} \beta_{2} \alpha_{5}$ form sandwich domains consisting of the metal binding site, the DXE motif; $\alpha_{6}$ and $\alpha_{7}$ form a long turnhelix-coil that connects the $\mathrm{N}$-terminal region and the central region. The central region contains a Rossmann-like fold $\left(\beta_{4} \alpha_{9} \beta_{5} \alpha_{10} \beta_{6}\right)$. The $\mathrm{C}$-terminal region is composed of a long coil region, a short helical turn $\left(\alpha_{12}\right)$ and a short $\beta$-strand $\left(\beta_{7}\right)$. The nucleotide-binding sites are located at the edge of the $\beta$-sheet ( $\beta 1$ and $\beta 6$ ), helices $\alpha 3$ and $\alpha 7$ and the $C$-terminal loop.

The DUF1792 structure is distinctly different from the two classic glycosyltransferase folds, GT-A and GT-B (Fig. 5d and e). In addition, it does not share any structural similarity with a previously suggested GT-C fold.

\section{The DUF1792 structure represents a new GT fold}

To date, numerous structures have been solved and reported for glycosyltransferases. Structural alignment of DUF1792 performed using the Secondary Structure Matching server $^{42}$ from the European Bioinformatics Institute revealed that the closest match is the class B nonspecific acid phosphatase (AphA protein, PDB entry 1Z5G) from Salmonella typhimurium, with a RMSD deviation of $4.7 \AA$. AphA has a haloacid dehalogenase-like fold that is conserved in members of the DDDD superfamily of phosphohydrolases ${ }^{43}$. Upon visual inspection, it is clear that only the so-called Rossmann-like fold of the DUF1792 structure superimposes onto the haloacid dehalogenase-like structure of AphA (Fig. 6a). A general search using the DALI server ${ }^{44}$ indicated that the closest match to DUF1792 in the database is 3-Dehydroquinate Synthase (DHQS) from Vibrio cholerae (PDB entry 3OKF) with a weak $\mathrm{Z}$ score of 4.8 and a RMS deviation of $3.3 \AA$ (Fig. $6 \mathrm{~b}$ ). DHQS catalyzes the formation of the first cyclic compound (3-Dehydroquinate) of the shikimate pathway, a promising target for the design of antimicrobial compounds ${ }^{45}$. DHQS requires NAD and a divalent cation to catalyze the reaction ${ }^{46}$. The similarity between DUF1792 and DHQS only extends to the Rossmann-like fold. In addition, there is no sequence homology among the three proteins. Key conserved residues important for glycosyltransferase activity among DUF1792 homologs (Fig. 3c) are not found in either 3-Dehydroquinate Synthase or AphA, further suggesting DUF1792 represents a new type of glycosyltransferase fold. Despite structures of a large number of glycosyltransferases from two well defined GT folds being resolved and documented ${ }^{8,9,10}$, the search failed to identify any structural homolog of DUF1792 from this large pool of reported glycosyltransferases and only revealed a weak homology to two non-glycosyltransferase enzymes, AphA and DHQS, further suggesting that the structure of DUF1792 is unique. Because this structure is distinct from two currently defined GT folds, GT-A and GT-B, and a previously designated GT-C fold ${ }^{39,} 40,15$, we have named it as the GT-D fold. 


\section{The GT-D fold possesses UDP and manganese binding sites}

The hallmark of glycosyltransferases is their ability to bind to nucleotide activated sugars ${ }^{47}$. Our biochemical assays revealed that UDP-glucose is the activated sugar used by DUF1792. In fact, during the crystallization of DUF1792, we found UDP-glucose is critical for recombinant DUF1792 to grow crystals. Presumably the structure of DUF1792 would require the presence of UDP-glucose. However, only UDP was found in the electron density map from DUF1792 (Fig. 5a, c and Fig. 7a), and there was no density for the glucose moiety from UDP-glucose, indicating that the glucose residue may be turned over by in-crystal catalysis. The same phenomenon has been observed for a number of glycosyltransferases using UDP-glucose $\mathrm{e}^{36,48}$ or other activated sugar donors ${ }^{49,50}$.

In nucleotide sugar binding sites, key amino acid residues with positive charges often interact with phosphate atoms. In DUF1792, the side chain of positively charged Arg28 interacts with both $a$ and $\beta$ phosphates of UDP at a distance of $2.8 \AA$ and $2.9 \AA$ (Fig. 7b). In addition, the Lys $205 \mathrm{~N} \varepsilon$ atom interacts with the a-phosphate of UDP at a distance of $3.05 \AA$. The phenyl rings of His 245 stack to the pentose sugar ribose of UDP (Fig. 7b) and His223 $\mathrm{N} \delta 1$ interacts with the $\mathrm{O} 3$ atom of the a phosphate (3.2A)(Fig. 7b). Similarly, a new epimerase responsible for synthesis of dTDP-L-rhamnose, RmlC, has two Arg residues which interact with the $\alpha$ - and $\beta$-phosphates of dTDP-phenol ${ }^{51,52}$. The first glycosyltransferase involved in biosynthesis of mycothiol of Corynebacterium glutamicum, MshA, has three Arg residues involved in the interaction with the phosphoesters of UDP ${ }^{53}$. These residues are crucial for activity of RmlC and MshA. Significantly, Arg28, His223 and His245 are conserved amino acid residues in DUF1792 homologs from both Gram-positive and Gram-negative bacteria (Fig. 3c). When Arg28 was mutated to Ala, the activity of the mutant DUF1792 (Arg28Ala) was completely inhibited. His223Ala and His245Ala mutants also exhibited much lower activity than the native DUF1792 (Fig. 7d), demonstrating that Arg28, His223 and His245 are indeed important for UDP binding, and that DUF1792 possesses a UDP-binding motif.

The DUF1792 domain is a metal dependent enzyme that requires bivalent metal ions for its activity (Fig. 2c). However, it does not have the typical metal binding motif, DXD, required for GT-A type glycosyltransferase ${ }^{54,55}$. Instead a DXE motif is found at residues 31 to 33 , albeit it does not directly interact with the manganese atom in the structure (Fig. 7c). In fact, when comparing all DUF1792 homologs from streptococci or even from Gram-negative bacteria, we found that the presence of the DXE motif was invariable and absolutely conserved (Fig. 3c). To define the requirement of a DXE motif for glycosyltransferase activity in DUF1792, we performed site-directed mutagenesis and mutated DXE to AXE or DXA. The activity was completely inhibited in these DUF1792 mutants. Furthermore, we mutated DXE to EXE or DXD, and observed a significant reduction in the enzyme activity of each mutant (Fig. 7d), demonstrating that even the conserved switch between $\mathrm{E}$ and $\mathrm{D}$ alters the glycosyltransferase activity of DUF1792. These results further suggest that the DXE motif is critical for the glycosyltransferase activity of DUF1792.

Close inspection of the structure revealed that the manganese ion bound at the active site of DUF1792 is octahedrally coordinated by only oxygen atoms: four water molecules, an 
oxygen atom from the $\beta$-phosphate of UDP, and one oxygen atom from the acetate ion most proximal to the UDP (Fig. 7c). Together, these results demonstrate that DUF1792 contains a metal binding (DXE) motif in this new family of glycosyltransferases.

\section{UDP and metal binding are crucial for in vivo glycosylation of Fap1}

To determine the requirement of UDP and metal binding sites in vivo in S. parasanguinis, we selected one key residue Asp31 engaged in the metal binding, and another one His223 involved in the UDP binding, to carry out site-directed mutagenesis and determined the impact of the mutated $d G T 1$ alleles on biogenesis of Fap1 in S. parasanguinis. D31A, D31E and H223A completely inhibited the production of mature Fap1 as determined by mature Fap1 specific mAbF51 antibody (Fig. 8a, lanes 5-7). The Fap1 variants generated by the $d G T 1$ site-directed mutants (Fig. 8b, lanes 5-7) show a similar migration pattern to the Fap1 protein from the $d G T 1$ non mutant (Fig. 8b, lane 3) when probed by Fap1-peptide specific antibody mAbE42, further demonstrating the importance of these two motifs in the glycosylation of Fap1 in vivo.

\section{DISCUSSION}

In this study we have defined a new glycosyltransferase superfamily, DUF1792, which is involved in the biosynthesis of bacterial O-glycans. Glycomic strategies have revealed that Fap1, a bacterial adhesin, is modified by a branched hexasaccharide with the sequence Rha1-3Glc $\beta 1$-(Glc1-3GlcNAc1-) 2,6 Glc $\beta 1-6$ GlcNAc. DUF1792 is a metal dependent betaglucosyltransferase, which transfers a Glc residue to the Glc-GlcNAc modified Fap1 at the branching point. The DUF1792 domain has a Rossmann-like nucleotide-binding fold, but does not show any sequence and structure identity with glycosyltransferases possessing currently annotated type GT-A or GT-B folds. The domain does not share any structural identity with a previously proposed GT-C fold either $39,40,15$. Moreover, DUF1792 has a highly conserved DXE motif instead of the classic DXD metal-binding motif found in the archetypical GT-A folds. Together our data lead us to propose that DUF1792 represents a new family of glycosyltransferases that display a unique glycosyltransferase fold which we have named GT-D.

The amino acid sequence constituting the fold is highly conserved in streptococci and even in Gram-negative bacteria. Our biochemical and structural studies of DUF1792 have not only defined the activity of this domain, but have also established a new family of bacterial glycosyltransferases with a previously uncharacterized GT fold. This GT-D fold is crucial for Fap1 biogenesis; moreover, the biogenesis of several Fap1-like proteins has been implicated in bacterial virulence ${ }^{30}$. Thus our characterization of this new GT-D type of glycosyltransferase may be helpful in guiding the design of antibacterial therapeutics targeting this activity.

\section{METHODS}

\section{Protein expression and purification}

The DUF1792 domain encoding amino acids 1-272 of dGT1 (AFJ26875) was amplified from genomic DNA of $S$. parasanguinis FW213 and cloned into the pET28a-sumo vector, 
and transformed to E. coli BL21 Gold (DE3) cells. All strains and primers used in this study were listed in Supplementary Table 2 and 3. The recombinant strain grown to $\mathrm{OD}_{600}=0.8$ in LB medium was then induced with $0.1 \mathrm{mM}$ IPTG at $18^{\circ} \mathrm{C}$ overnight. Native DUF1792 protein was purified with Histrap Column (Ni affinity) and gel filtration as described ${ }^{36}$. In brief, the overnight grown $E$. coli cells were harvested by centrifugation and lysed by sonication in binding buffer $(20 \mathrm{mM}$ Tris- $\mathrm{HCl} \mathrm{pH} \mathrm{8.0,500} \mathrm{mM} \mathrm{NaCl}$, and $25 \mathrm{mM}$ Imidazole). The clear cell lysates obtained after centrifugation (16, $500 \mathrm{rpm}$ for $1 \mathrm{~h}$ ) were subjected to protein purification using HiTrapTM Column $\left(\mathrm{Ni}^{2+} \_\right.$affinity). Proteins were eluted from the affinity resin by elution buffer $(20 \mathrm{mM}$ Tris- $\mathrm{HCl}, \mathrm{pH} 8.0,500 \mathrm{mM} \mathrm{NaCl}$, and $500 \mathrm{mM}$ imidazole). The N-terminal His-SUMO tag was cleaved by incubating the elution fractions with SUMO protease, ULP (ubiquitin-like protein protease), during overnight dialysis at $4^{\circ} \mathrm{C}$ against $20 \mathrm{mM}$ Tris- $\mathrm{HCl}, \mathrm{pH} 8.0,500 \mathrm{mM} \mathrm{NaCl}$. Dialyzed protein samples were reapplied to HiTrapTM Column to remove ULP, the cleaved His tag, and uncleaved proteins. Flow-through was collected and further purified by using a 16/60 Superdex 75 gel filtration column (GE Healthcare) with gel filtration buffer (20 mM Tris pH $8.0,100 \mathrm{mM} \mathrm{NaCl}$ and $1 \mathrm{mM}$ DTT). Protein purity was analyzed by SDS-PAGE. Peak fractions were collected and concentrated to $30 \mathrm{mg}$ per $\mathrm{ml}$ for crystallization screen. Selenomethionyl DUF1792 was obtained by growing the recombinant strain in M9 medium with selenomethionine at $60 \mathrm{mg}$ per $\mathrm{L}^{56}$. The selenomethionyl protein was purified using the same protocol as the native protein.

\section{Crystallization, data collection and refinement}

The purified protein was concentrated to $10 \mathrm{mg}$ per $\mathrm{ml}$ in $20 \mathrm{mMTris}$ buffer (pH 8.0), 100 $\mathrm{mM} \mathrm{NaCl}, 1 \mathrm{mM}$ DTT and subjected to crystallization trials. Hanging-drop vapor-diffusion method was used for the crystallization trials. Crystals were obtained in the following solution. $1 \mu \mathrm{L}$ protein solution (10 mg per $\mathrm{mL}$ ) with $10 \mathrm{mM}$ UDP-Glc was mixed with $1 \mu \mathrm{L}$ well solution (500 uL) consisting of $100 \mathrm{mM}$ Tris buffer (pH 8.5), 35\% PEG 1500, $200 \mathrm{mM}$ Sodium Acetate. A single crystal was cryo-cooled in liquid nitrogen after being cryo protected by addition of 10\% glycerol. The Se-Met DUF1792 and native DUF1792 in the complex with Mn were crystallized utilizing the same conditions. All DUF1792 crystals appeared in three days.

Data were collected with an oscillation angle of $1^{\circ}$ per image on beamline SER-CAT ID 22 at the Argonne National Laboratory. The structure was determined by multiwavelength anomalous dispersion ${ }^{15}$, utilizing Se atoms as the anomalous scatterers. Se-Met-DUF1792 data were collected at wavelength of 0.97877 (Peak), 0.97907 (Edge) and 0.97142 (High). Data of native-DUF1792 and DUF1792-Mn were collected at wavelength of 1.00000. All data were collected under temperature $100 \mathrm{~K}$. Data were processed and scaled with HKL2000 57 . The model building and subsequent structure refinement were performed with the Phenix software ${ }^{58}$. Restrained individual B-factor and TLS refinement were not performed until the last cycle. After each cycle of refinement, the model was manually rebuilt based on the resulting $2 \mathrm{~F}_{\mathrm{o}}-\mathrm{F}_{\mathrm{c}}$ and $\mathrm{F}_{\mathrm{o}}-\mathrm{F}_{\mathrm{c}}$ maps. 


\section{Expression and purification of rFap1-RI}

We used rFap1-RI, a small fragment of Fap1 that contains the first repeat region (100 to 200 amino acid residues of Fap1), as a model to carry out glycan profiling study of Fap1. Unglycosylated rFap1-R1 was purified from a recombinant strain that carries pET28arFap1-R1. Glycosylated Fap1-R1 was purified from a recombinant BL21 strain that was constructed by coexpression of pET28a-rFap1-R1 with pVPT-Gtf123-dGT1-GalT2 and pHSG576-Gly, in which glycosylated rFap1-R1 was modified by all putative glycosyltransferase, Gtf12, Gtf3, dGT1, GalT2 and Gly. The plasmids and strains were constructed as described in Supplementary Table 2. All plasmids were confirmed by sequencing. Unglycosylated and glycosylated rFap1-RI were purified using the same method as recombinant DUF1792 protein.

\section{Glycan profiling of modified rFap1-R1}

Purified rFap1-R1 was subjected to reductive elimination and permethylation as described below.

\section{Reductive Elimination}

The glycans were reductively eliminated from $\mathrm{rFap} 1$ and purified on a $50 \mathrm{E}-8 \mathrm{C}$ Dowex ${ }^{\circledR}$ column as previously described ${ }^{59}$, and the purified glycans were subjected to permethylation and purified according to published methods. Briefly, the freeze-dried rFap1 sample was dissolved in $55 \mathrm{mg}$ per $\mathrm{mL}$ potassium borohydride in a $1 \mathrm{~mL}$ of a $0.1 \mathrm{M}$ potassium hydroxide solution. The mixture was incubated at $45^{\circ} \mathrm{C}$ for $18 \mathrm{hrs}$ and quenched by adding 5-6 drops of acetic acid. The sample was loaded onto the Dowex ${ }^{\circledR}$ column and eluted with $5 \%$ acetic acid. The collected solution was concentrated and lyophilized. Excessive borates were removed with $10 \%$ methanolic acetic acid.

\section{Permethylation}

For the permethylation reaction, sodium hydroxide (3-5 pellets per sample) was crushed in $3 \mathrm{~mL}$ dry dimethyl sulfoxide. The resulting slurry $(0.75 \mathrm{~mL})$ and methyl iodine $(500 \mu \mathrm{L})$ were added to the sample. The mixture was agitated for $15 \mathrm{~min}$ and quenched by adding 2 $\mathrm{mL}$ ultra-pure water with shaking. The glycans were extracted with chloroform $(2 \mathrm{~mL})$ and washed with ultra-pure water two times. Chloroform was removed under a stream of nitrogen. The permethylated glycans were loaded onto a C18 Sep-pak® column, washed with $5 \mathrm{~mL}$ ultra-pure water, and successively eluted with $3 \mathrm{~mL}$ each of $15 \%, 35 \%, 50 \%$ and $75 \%$ aq. acetonitrile. The solutions were collected and lyophilized.

\section{Peracetylation}

A previously described method was used for peracetylation ${ }^{60}$. Glycans were incubated with $200 \mu \mathrm{L}$ pyridine and $200 \mu \mathrm{L}$ acetic anhydride at $80^{\circ} \mathrm{C}$ for $3 \mathrm{hrs}$, after which the reagent was removed under a stream of nitrogen. The acetylated glycans were dissolved in chloroform and washed 3 times with pure water, and the chloroform was removed under a stream of nitrogen. 


\section{$\mathrm{CrO}_{3}$ oxidation}

$10 \mathrm{mg} \mathrm{CrO}_{3}$ was added to $100 \mu \mathrm{L}$ of acetic acid. The slurry was added to peracetylated samples, and the mixture was heated to $50{ }^{\circ} \mathrm{C}$ and kept for $3 \mathrm{hrs}$. After quenching the reaction with water, the product of oxidation was extracted with chloroform and washed with water twice.

\section{Mass Spectrometry}

MS data were acquired by using either a Voyager DE-STRTM MALDI-TOF or a 4800 MALDI-TOF/TOF mass spectrometer (Applied Biosystems, Darmstadt, Germany). MS/MS data were acquired with the latter instrument. MS mode was calibrated with 4700 Calibration standard kit (Applied Biosystems), and MS/MS mode was calibrated with fibrinopeptide B human (Sigma). For MS/MS studies, the collision energy was set to $1 \mathrm{kV}$, and the collision gas was argon. 2, 5-dihydroxybenzoic acid was used as matrix. Permethylated samples were dissolved in $10 \mu \mathrm{L}$ methanol, $1 \mu \mathrm{L}$ of this solution was premixed with $1 \mu \mathrm{L}$ matrix and $1 \mu \mathrm{L}$ of the mixture was spotted onto the plate.

\section{GC-MS Trimethylsilyl (TMS) analysis}

The glycan sample was incubated with $1.0 \mathrm{M}$ methanolic $\mathrm{HCl}$ at $80{ }^{\circ} \mathrm{C}$ for $14 \mathrm{hrs}$. The reagent was removed under a stream of nitrogen. $500 \mu \mathrm{L}$ methanol, $10 \mu \mathrm{L}$ pyridine and 50 $\mu \mathrm{L}$ acetic anhydride and were successively added to the sample. The mixture was kept at room temperature for $15 \mathrm{~min}$ and the reagent was removed under a stream of nitrogen. 200 $\mu \mathrm{L}$ Tri-Sil $\mathrm{Z}$ reagent was added to the sample, and the mixture was kept at room temperature for $15 \mathrm{~min}$. After removing the reagent under a stream of nitrogen, the sample was washed by hexane twice. A PerkinElmer Clarus 500 instrument fitted with a RTX-5 fused silica capillary column was used for carrying out the analysis. The following temperature programme was used for eluting the sample. The oven temperature was initially $65^{\circ} \mathrm{C}$, and heated to $140{ }^{\circ} \mathrm{C}$ at the rate of $25^{\circ} \mathrm{C}$ per min, and heated to $200{ }^{\circ} \mathrm{C}$ at the rate of $5{ }^{\circ} \mathrm{C}$ per min. The temperature was finally raised to $300^{\circ} \mathrm{C}$ at a rate of $10{ }^{\circ} \mathrm{C}$ per min and is held for 5 $\min$.

\section{GC-MS Linkage Analysis}

Partially methylated alditol acetates were prepared as previously described ${ }^{61}$. A PerkinElmer Clarus 500 instrument fitted with a RTX-5 fused silica capillary column was used for carrying out linkage analysis. A linear gradient temperature programme was used: the sample was injected into the column at $60{ }^{\circ} \mathrm{C}$, and the temperature increases to $300^{\circ} \mathrm{C}$ over $30 \mathrm{~min}$ at a rate of $8{ }^{\circ} \mathrm{C}$ per min.

\section{Construct of a dgT1 knock-out mutant}

A non-polar d $g T l$ knock-out mutant was generated by insertional mutagenesis with a kanamycin resistance cassette $\left(\operatorname{Kan}^{\mathrm{r}}\right)$. Briefly, the $\mathrm{d} g T 1$ gene and its flanking regions including the 600-bp upstream and 600-bp downstream regions were amplified from genomic DNA of $S$. parasanguinis FW213. The PCR fragment was purified and cloned into pGEM-T Easy vector (Promega, Madison, WI). A 1500-bp dgTl internal fragment was replaced with an 830-bp nonpolar kanamycin resistance cassette (aphA3) isolated from 
pALH1 $24^{62}$ by an inverse PCR strategy. Plasmid was confirmed by sequencing, and then transformed into the FW213 strain by electroporation. The transformants were selected on $\mathrm{TH}$ agar plates containing kanamycin. The $\mathrm{d} g \mathrm{Tl}$ allelic replacement mutant was selected by its ability to resist kanamycin and its susceptibility to tetracycline, and was further verified by PCR and sequencing analysis. The confirmed $\mathrm{d} G T 1$ allelic replacement mutant was used in this study.

\section{Western blot analysis}

For all S. parasanguinis strains, bacteria grown to an optical density at $470 \mathrm{~nm}$ (OD470) of 0.5 to 0.6 were harvested by centrifugation. The cell pellets were treated with amidase to lyse the cells ${ }^{31}$. For $E$. coli strains, bacteria grown to an optical density at 600nm (OD600) of 0.6 to 0.7 were harvested by centrifugation. Cell lysates were prepared by boiling the cell pallets collected in sample buffer $(0.0625 \mathrm{M}$ Tris $\mathrm{HCl}$ [pH 6.8], $2 \%$ sodium dodecyl sulfate [SDS], $10 \%$ glycerol, $0.01 \%$ bromophenol blue) for $10 \mathrm{~min}$ before being loaded onto $8 \%$ SDS-polyacrylamide gel electrophoresis (PAGE) gels and subjected to Western blotting. Two monoclonal anti-mouse antibodies (MAbs) were used to detect Fap1: MAb E42 (1:3000), which is specific to the peptide backbone of Fap1, and MAb F51(1:5000), which is specific to mature Fap ${ }^{63}$. A polyclonal anti-rabbit antibody against DNAK (a gift from José Lemos at University of Rochester) was used to standardize the protein loading of $S$. parasanguinis lysates. All western blot figures shown in this paper were cropped. Uncropped figures are supplied in Supplementary Figure 4.

\section{Site-direct mutagenesis, in vitro and in vivo Fap1 glycosylation}

Site-direct mutagenesis was carried out using QuickChange mutagenesis kit (Stratagene) as described $^{36}$. The plasmid pET28a-sumo-DUF1792 and pVPT-dGT1 were used as templates. Mutant constructs were identified and confirmed by sequencing. Mutated DUF1792 proteins were purified using the same method as described above. Gtf1/2,3 modified rFap1 was purified using Glutathione Sepharose 4B beads according to the manufacturer's protocol (Amersham) and used as a substrate for the in vitro glycosyltransferase assays as described previously ${ }^{35}$. In brief, the substrate and enzyme bound to glutathione-Sepharose beads were washed five times with glycosylation buffer (50 mM Hepes, $\mathrm{pH} 7.0,10 \mathrm{mM} \mathrm{MnCl} 2,0.01 \%$ bovine serum albumin). $20 \mu \mathrm{g}$ of recombinantdGT1 or its variants and substrate Fap1 were mixed with $0.2 \mu \mathrm{Ci}$ of UDP-[3H]glucose $(28 \mathrm{Ci} / \mathrm{mmol}$; Amersham Biosciences) or $0.2 \mu \mathrm{Ci}$ of UDP-[3H]GlcNAc (2.8 Ci/mmol; Amersham Biosciences) in a final volume of $200 \mu \mathrm{L}$ of glycosylation buffer and incubated for $1 \mathrm{~h}$ at $37^{\circ} \mathrm{C}$. The beads in the glycosylation assays were washed three times with NETN buffer ( $20 \mathrm{mM}$ Tris-HCl, $100 \mathrm{mM} \mathrm{NaCl}, 1 \mathrm{mM}$ EDTA, $0.2 \% \mathrm{NP} 40, \mathrm{pH} 7.0$ ) and then transferred to scintillation vials to measure radioactivity transferred to the Fap1 substrates from the radiolabeled activated sugars. The assays were performed in triplicate in three independent experiments.

DUF1792 homologs from S. agalactiae J48, S. pneumonia TIGR4, S. sanguinis SK36 and Fusobacterium nucleatum were amplified from each strain and cloned to pGEX-6p-1 to generate pGEX-DUF1792 respectively. pHSG576-rFap1 (pAL80), pVPT-gtf1-2-3 and pGEX-DUF1792 were co-transformed into E. coli. The ability of DUF1792 homologs to 
glycosylate Gtf1/2, 3-modified rFap1 was examined using Western blotting analysis with Fap1 peptide-specific monoclonal antibody E42 at 1:3000 dilution.

The construct pVPT- $d G T 1$, pVPT-DUF1792, pVPT-dGT1-Cterminus and site-directed mutants of $d G T 1$ variants were used to transform the $\mathrm{d} G T 1$ knockout to determine the effect of site-directed mutations within this DUF1792domain on Fap1 glycosylation in vivo. Biogenesis of Fap1 was detected by Western blotting analysis using the Fap1-specific monoclonal antibodies E42 (peptide-specific) and F51 (mature Fap1).

\section{Statistics}

The two-tailed Student's t-test was used to determine statistically significant differences between groups and statistically significant differences with p-values below 0.006 are indicated with two asterisk $(* *)$ and below 0.0006 are indicated with three asterisk $(* * *)$.

\section{Supplementary Material}

Refer to Web version on PubMed Central for supplementary material.

\section{Acknowledgments}

This study was supported by NIH/NIDCR F33DE022215 and R01DE017954 (H. Wu) and the Biotechnology and Biological Sciences Research Council (AD and SMH).

\section{References}

1. Nothaft H, Szymanski CM. Protein glycosylation in bacteria: sweeter than ever. Nature Reviews Microbiology. 2010; 8:765-778. [PubMed: 20948550]

2. Schwarz F, Aebi M. Mechanisms and principles of N-linked protein glycosylation. Current Opinion in Structural Biology. 2011; 21:576-582. [PubMed: 21978957]

3. Fletcher CM, Coyne MJ, Villa OF, Chatzidaki-Livanis M, Comstock LE. A general O-glycosylation system important to the physiology of a major human intestinal symbiont. Cell. 2009; 137:321-331. [PubMed: 19379697]

4. Ohtsubo K, Marth JD. Glycosylation in cellular mechanisms of health and disease. Cell. 2006; 126:855-867. [PubMed: 16959566]

5. Varki A. Nothing in glycobiology makes sense, except in the light of evolution. Cell. 2006; 126:841-845. [PubMed: 16959563]

6. Campbell J, Davies G, Bulone V, Henrissat B. A classification of nucleotide-diphospho-sugar glycosyltransferases based on amino acid sequence similarities. Biochemical Journal. 1997; 326:929. [PubMed: 9334165]

7. Drickamer K, Taylor ME. Evolving views of protein glycosylation. Trends in Biochemical Sciences. 1998; 23:321-324. [PubMed: 9787635]

8. Lairson L, Henrissat B, Davies G, Withers S. Glycosyltransferases: structures, functions, and mechanisms. Biochemistry. 2008; 77:521.

9. Liu J, Mushegian A. Three monophyletic superfamilies account for the majority of the known glycosyltransferases. Protein Science. 2003; 12:1418-1431. [PubMed: 12824488]

10. Coutinho PM, Deleury E, Davies GJ, Henrissat B. An evolving hierarchical family classification for glycosyltransferases. Journal of Molecular Biology. 2003; 328:307-317. [PubMed: 12691742]

11. Busch C, Hofmann F, Selzer J, Munro S, Jeckel D, Aktories K. A common motif of eukaryotic glycosyltransferases is essential for the enzyme activity of large clostridial cytotoxins. Journal of Biological Chemistry. 1998; 273:19566-19572. [PubMed: 9677381] 
12. Charnock SJ, Davies GJ. Structure of the nucleotide-diphospho-sugar transferase, SpsA from Bacillus subtilis, in native and nucleotide-complexed forms. Biochemistry. 1999; 38:6380-6385. [PubMed: 10350455]

13. Vrielink A, Rüger W, Driessen H, Freemont PS. Crystal structure of the DNA modifying enzyme beta-glucosyltransferase in the presence and absence of the substrate uridine diphosphoglucose. The EMBO Journal. 1994; 13:3413. [PubMed: 8062817]

14. Moréra S, et al. High resolution crystal structures of T4 phage [beta]-glucosyltransferase: induced fit and effect of substrate and metal binding1. Journal of Molecular Biology. 2001; 311:569-577. [PubMed: 11493010]

15. Igura M, et al. Structure-guided identification of a new catalytic motif of oligosaccharyltransferase. The EMBO Journal. 2007; 27:234-243. [PubMed: 18046457]

16. Lovering AL, De Castro LH, Lim D, Strynadka NCJ. Structural insight into the transglycosylation step of bacterial cell-wall biosynthesis. Science. 2007; 315:1402. [PubMed: 17347437]

17. Yuan Y, Barrett D, Zhang Y, Kahne D, Sliz P, Walker S. Crystal structure of a peptidoglycan glycosyltransferase suggests a model for processive glycan chain synthesis. Proceedings of the National Academy of Sciences of the United States of America. 2007; 104:5348. [PubMed: 17360321]

18. Froeliger EH, Fives-Taylor P. Streptococcus parasanguis fimbria-associated adhesin Fap1 is required for biofilm formation. Infection and Immunity. 2001; 69:2512. [PubMed: 11254614]

19. Mistou MY, Dramsi S, Brega S, Poyart C, Trieu-Cuot P. Molecular dissection of the secA2 locus of group B Streptococcus reveals that glycosylation of the Srr1 LPXTG protein is required for full virulence. Journal of Bacteriology. 2009; 191:4195. [PubMed: 19395494]

20. Sanchez CJ, et al. The pneumococcal serine-rich repeat protein is an intra-species bacterial adhesin that promotes bacterial aggregation in vivo and in biofilms. PLoS Pathogens. 2010; 6:e1001044. [PubMed: 20714350]

21. Wu H, Mintz KP, Ladha M, Fives-Taylor PM. Isolation and characterization of Fap1, a fimbriaeassociated adhesin of Streptococcus parasanguis FW213. Molecular Microbiology. 1998; 28:487500. [PubMed: 9632253]

22. Bu S, et al. Interaction between two putative glycosyltransferases is required for glycosylation of a serine-rich streptococcal adhesin. Journal of Bacteriology. 2008; 190:1256-1266. [PubMed: 18083807]

23. Wu H, Fives-Taylor PM. Identification of dipeptide repeats and a cell wall sorting signal in the fimbriae-associated adhesin, Fap1, of Streptococcus parasanguis. Molecular Microbiology. 1999; 34:1070-1081. [PubMed: 10594831]

24. Bensing BA, Sullam PM. An accessory sec locus of Streptococcus gordonii is required for export of the surface protein GspB and for normal levels of binding to human platelets. Molecular Microbiology. 2002; 44:1081-1094. [PubMed: 12010500]

25. Xu P, et al. Genome of the opportunistic pathogen Streptococcus sanguinis. Journal of Bacteriology. 2007; 189:3166. [PubMed: 17277061]

26. Seifert KN, Adderson EE, Whiting AA, Bohnsack JF, Crowley PJ, Brady LJ. A unique serine-rich repeat protein (Srr-2) and novel surface antigen $(\varepsilon)$ associated with a virulent lineage of serotype III Streptococcus agalactiae. Microbiology. 2006; 152:1029. [PubMed: 16549667]

27. Zhang YQ, et al. Genome-based analysis of virulence genes in a non-biofilm-forming Staphylococcus epidermidis strain (ATCC 12228). Molecular Microbiology. 2003; 49:1577-1593. [PubMed: 12950922]

28. Takeuchi F, et al. Whole-genome sequencing of Staphylococcus haemolyticus uncovers the extreme plasticity of its genome and the evolution of human-colonizing staphylococcal species. Journal of Bacteriology. 2005; 187:7292-7308. [PubMed: 16237012]

29. Siboo IR, Chaffin DO, Rubens CE, Sullam PM. Characterization of the accessory Sec system of Staphylococcus aureus. Journal of Bacteriology. 2008; 190:6188. [PubMed: 18621893]

30. Zhou M, Wu H. Glycosylation and biogenesis of a family of serine-rich bacterial adhesins. Microbiology. 2009; 155:317. [PubMed: 19202081] 
31. Wu H, Bu S, Newell P, Chen Q, Fives-Taylor P. Two gene determinants are differentially involved in the biogenesis of Fap1 precursors in Streptococcus parasanguis. Journal of Bacteriology. 2007; 189:1390. [PubMed: 16997950]

32. Wu H, Zeng M, Fives-Taylor P. The glycan moieties and the N-terminal polypeptide backbone of a fimbria-associated adhesin, Fap1, play distinct roles in the biofilm development of Streptococcus parasanguinis. Infection and Immunity. 2007; 75:2181-2188. [PubMed: 17296746]

33. Wu R, Wu H. A Molecular Chaperone Mediates a Two-protein Enzyme Complex and Glycosylation of Serine-rich Streptococcal Adhesins. Journal of Biological Chemistry. 2011; 286:34923-34931. [PubMed: 21862581]

34. Wu R, Zhou M, Wu H. Purification and characterization of an active Nacetylglucosaminyltransferase enzyme complex from Streptococci. Applied and Environmental Microbiology. 2010; 76:7966-7971. [PubMed: 20971868]

35. Zhou M, Zhu F, Dong S, Pritchard DG, Wu H. A novel glucosyltransferase is required for glycosylation of a serine-rich adhesin and biofilm formation by Streptococcus parasanguinis. Journal of Biological Chemistry. 2010; 285:12140. [PubMed: 20164186]

36. Zhu F, et al. Structural and functional analysis of a new subfamily of glycosyltransferases required for glycosylation of serine-rich streptococcal adhesins. Journal of Biological Chemistry. 2011; 286:27048-27057. [PubMed: 21653318]

37. Forde BM, et al. Genome sequences and comparative genomics of two Lactobacillus ruminis strains from the bovine and human intestinal tracts. Microbial Cell Factories. 2011; 10:S13. [PubMed: 21995554]

38. Kuwahara T, et al. Genomic analysis of Bacteroides fragilis reveals extensive DNA inversions regulating cell surface adaptation. Proceedings of the National Academy of Sciences of the United States of America. 2004; 101:14919. [PubMed: 15466707]

39. Takahashi M, et al. PIG-B, a membrane protein of the endoplasmic reticulum with a large lumenal domain, is involved in transferring the third mannose of the GPI anchor. The EMBO Journal. 1996; 15:4254. [PubMed: 8861954]

40. Maeda Y, et al. PIG-M transfers the first mannose to glycosylphosphatidylinositol on the lumenal side of the ER. The EMBO Journal. 2001; 20:250-261. [PubMed: 11226175]

41. Khoo K-H, Dell A. Assignment of anomeric configurations of pyranose sugars in oligosaccharides using a sensitive FAB-MS strategy. Glycobiology. 1990; 1:83-91. [PubMed: 2136384]

42. Krissinel E, Henrick K. Secondary-structure matching (SSM), a new tool for fast protein structure alignment in three dimensions. Acta crystallographica Section D, Biological crystallography. 2004; 60:2256-2268.

43. Makde RD, Gupta GD, Mahajan SK, Kumar V. Structural and mutational analyses reveal the functional role of active-site Lys-154 and Asp-173 of Salmonella typhimurium AphA protein. Archives of Biochemistry and Biophysics. 2007; 464:70-79. [PubMed: 17570338]

44. Holm L, Rosenstrom P. Dali server: conservation mapping in 3D. Nucleic acids research. 2010; 38:W545-549. [PubMed: 20457744]

45. Hawkins AR, Lamb HK. The Molecular Biology of Multidomain Proteins Selected Examples. European Journal of Biochemistry. 1995; 232:7-18. [PubMed: 7556173]

46. Barten R, Meyer T. Cloning and characterisation of the Neisseria gonorrhoeaearoB gene. Molecular and General Genetics. 1998; 258:34-44. [PubMed: 9613570]

47. Breton C, Imberty A. Structure/function studies of glycosyltransferases. Current Opinion in Structural Biology. 1999; 9:563-571. [PubMed: 10508766]

48. Jinek M, Chen YW, Clausen H, Cohen SM, Conti E. Structural insights into the Notch-modifying glycosyltransferase Fringe. Nature Structural and Molecular Biology. 2006; 13:945-946.

49. Chiu CPC, et al. Structural analysis of the sialyltransferase CstII from Campylobacter jejuni in complex with a substrate analog. Nature Structural and Molecular Biology. 2004; 11:163-170.

50. Chang A, et al. Complete set of glycosyltransferase structures in the calicheamicin biosynthetic pathway reveals the origin of regiospecificity. Proceedings of the National Academy of Sciences of the United States of America. 2011; 108:17649-17654. [PubMed: 21987796] 
51. Giraud MF, Leonard GA, Field RA, Berlind C, Naismith JH. RmlC, the third enzyme of dTDP-Lrhamnose pathway, is a new class of epimerase. Nature Structural \& Molecular Biology. 2000; 7:398-402.

52. Ramakrishnan B, Boeggeman E, Ramasamy V, Qasba PK. Structure and catalytic cycle of [beta]-1, 4-galactosyltransferase. Current Opinion in Structural Biology. 2004; 14:593-600. [PubMed: 15465321]

53. Vetting MW, Frantom PA, Blanchard JS. Structural and Enzymatic Analysis of MshA from Corynebacterium glutamicum. Journal of Biological Chemistry. 2008; 283:15834-15844. [PubMed: 18390549]

54. Tarbouriech N, Charnock SJ, Davies GJ. Three-dimensional structures of the Mn and Mg dTDP complexes of the family GT-2 glycosyltransferase SpsA: a comparison with related NDP-sugar glycosyltransferases1. Journal of Molecular Biology. 2001; 314:655-661. [PubMed: 11733986]

55. Persson K, Ly HD, Dieckelmann M, Wakarchuk WW, Withers SG, Strynadka NCJ. Crystal structure of the retaining galactosyltransferase LgtC from Neisseria meningitidis in complex with donor and acceptor sugar analogs. Nature Structural and Molecular Biology. 2001; 8:166-175.

56. Doublié S. Preparation of selenomethionyl proteins for phase determination. Methods in Enzymology. 1997; 276:523-530. [PubMed: 9048379]

57. Otwinowski Z, Minor W. Processing of X-ray diffraction data collected in oscillation mode. Methods in Enzymology. 1997; 276:307-326.

58. Adams PD, et al. PHENIX: a comprehensive Python-based system for macromolecular structure solution. Acta crystallographica Section D, Biological crystallography. 2010; 66:213-221.

59. North SJ, et al. Chapter Two-Mass Spectrometric Analysis of Mutant Mice. Methods in Enzymology. 2010; 478:27-77. [PubMed: 20816474]

60. Haslam SM, Khoo K-H, Houston KM, Harnett W, Morris HR, Dell A. Characterisation of the phosphorylcholine-containing N-linked oligosaccharides in the excretory-secretory $62 \mathrm{kDa}$ glycoprotein ofAcanthocheilonema viteae. Molecular and biochemical parasitology. 1997; 85:5366. [PubMed: 9108548]

61. Jang-Lee J, et al. Glycomic profiling of cells and tissues by mass spectrometry: fingerprinting and sequencing methodologies. Methods in Enzymology. 2006; 415:59-86. [PubMed: 17116468]

62. Kremer BH, van der Kraan M, Crowley PJ, Hamilton IR, Brady LJ, Bleiweis AS. Characterization of the sat operon in Streptococcus mutans: evidence for a role of Ffh in acid tolerance. Journal of Bacteriology. 2001; 183:2543-2552. [PubMed: 11274114]

63. Stephenson AE, Wu H, Novak J, Tomana M, Mintz K, Fives-Taylor P. The Fap1 fimbrial adhesin is a glycoprotein: antibodies specific for the glycan moiety block the adhesion of Streptococcus parasanguis in an in vitro tooth model. Mol Microbiol. 2002; 43:147-157. [PubMed: 11849543] 
a

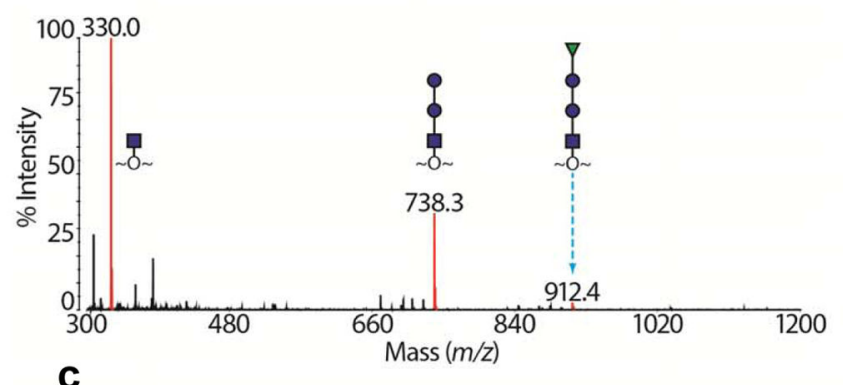

C

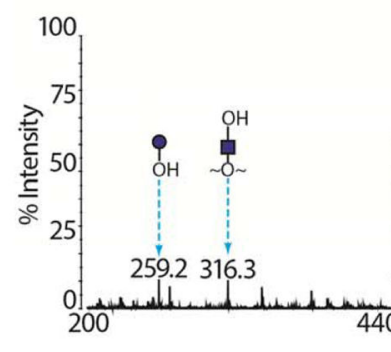

b
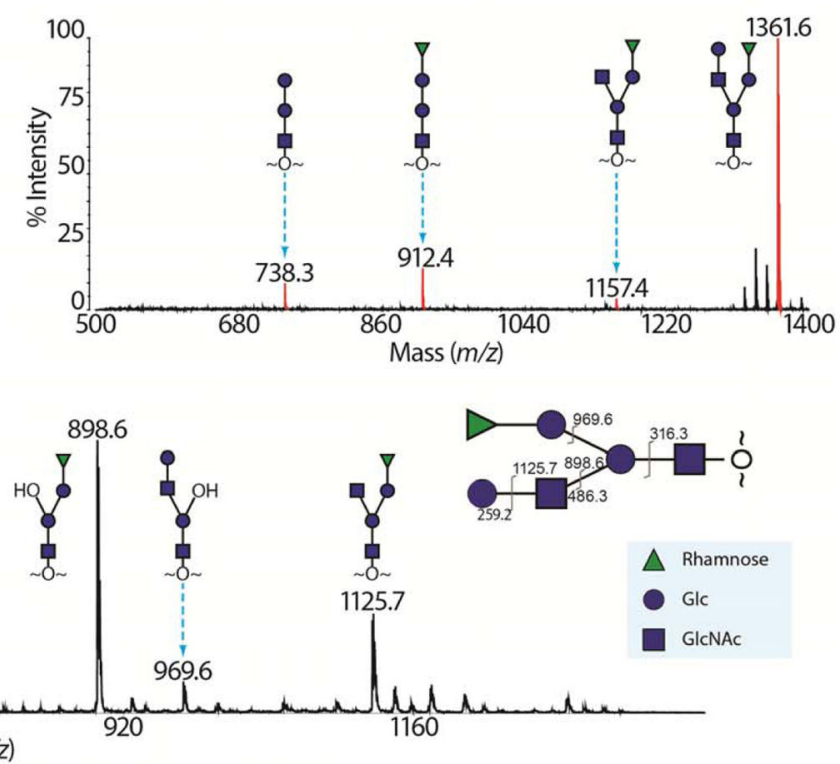

Figure 1. Mass spectrometric analysis of rFap1 glycan

The glycans were reductively eliminated from the protein, and permethylated.

Derivatized glycans were purified on reverse phase C18 Sep-Pak ${ }^{\circledR}$ columns. The MALDITOF spectra of the 35\% acetonitrile (MeCN) eluate (a), the 50\% MeCN eluate (b), and MALDI-TOF/TOF spectrum of the peak at mass-to-charge ratio $(\mathrm{m} / \mathrm{z}) 1361.6(\mathrm{c})$ are shown. In spectra (a) and (b), peaks corresponding to sodiated glycans are colored red and annotated with $m / z$ and glycan structures. Other black signals are due to under-permethylation (minus 14 in $\mathrm{m} / \mathrm{z}$ ) or contaminations from the matrix. In spectra (c), peaks corresponding to diagnostic fragments are annotated with $m / z$ and glycan structure. Notably, the peak at $m / z$ 506.4 corresponds to a double-cleaved fragment, indicating the fully synthesized glycan is branched. 
a

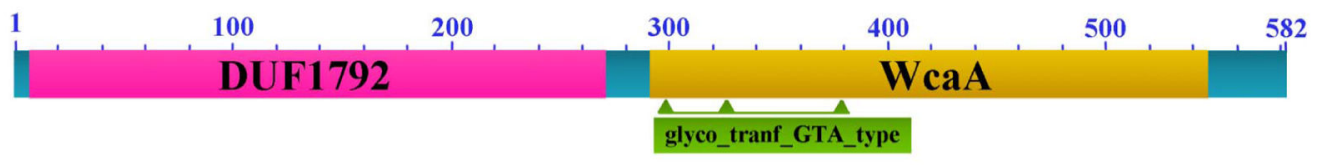

b

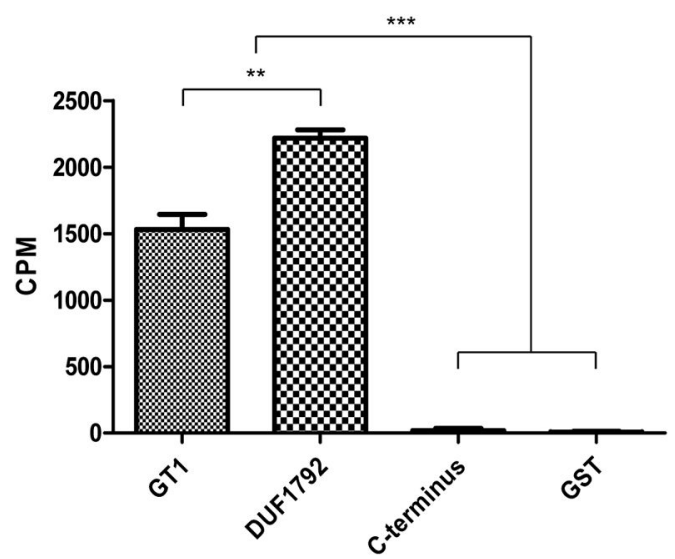

C

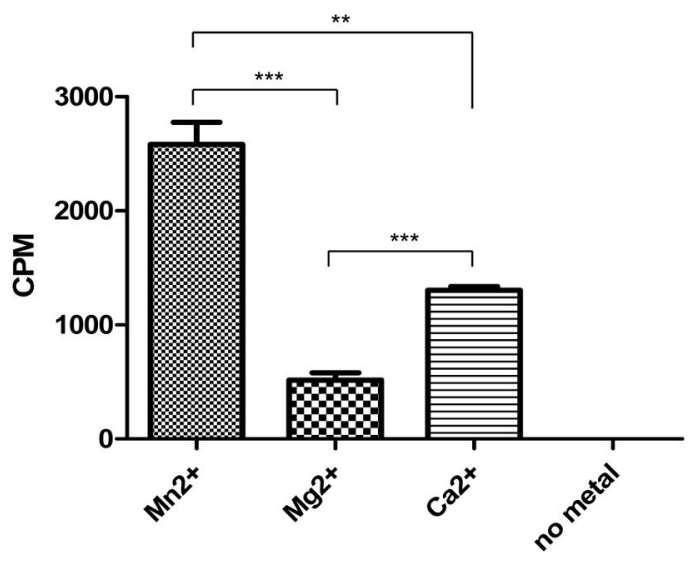

Figure 2. Glycosyltransferase activity of DUF1792

(a) Diagram of dGT1. dGT1 consists of two domains, an N-terminal DUF1792 and a Cterminal GT-A-like domain (WcaA, glycosyltransferase involved in cell wall biosynthesis). (b) In vitro glycosyltransferase activity of DUF1792. Purified dGIT1, and N-terminal DUF1792 fusion proteins catalyzed the transfer of ${ }^{3} \mathrm{H}$ labeled glucose from an activated donor sugar, UDP- ${ }^{3} \mathrm{H}$ glucose to Gtf1/2,3-modified Fap1 in in vitro glycosylation reactions, while the C-terminus of dGT1 GST fusion protein and GST failed to transfer. Heated inactivated proteins were used as negative controls to normalize the transfer activity by CPM.

(c) Metal ions are required for glycosyltransferase activity of DUF1792. Divalent metal ions, $\mathrm{Mn}^{2+}, \mathrm{Mg}^{2+}$ and $\mathrm{Ca}^{+}(10 \mathrm{mM})$ promoted the transfer of ${ }^{3} \mathrm{H}$ labeled glucose from an activated donor sugar, UDP- ${ }^{3} \mathrm{Hglucose}$ to Gtf1/2, 3-modified Fap1 in in vitro glycosylation reactions. The value obtained from three different experiments represent means \pm standard errors of the means. Significant differences were indicated by asterisks $(* * P<0.01$, *** $P<0.001)$. 


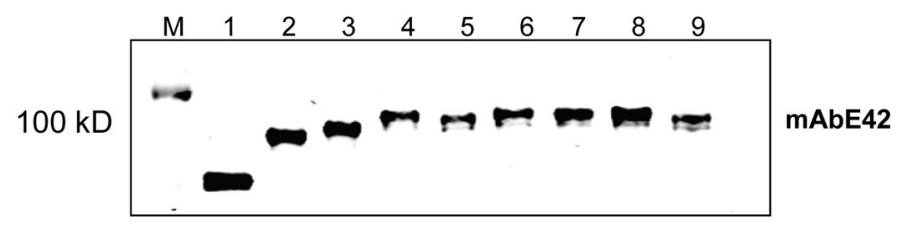

b

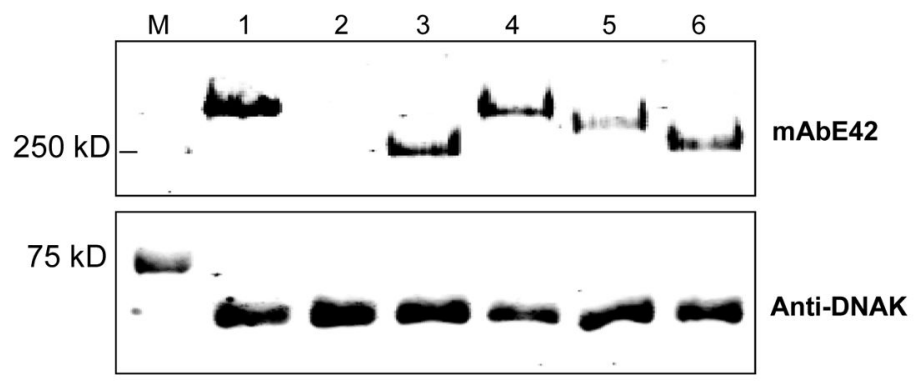

C

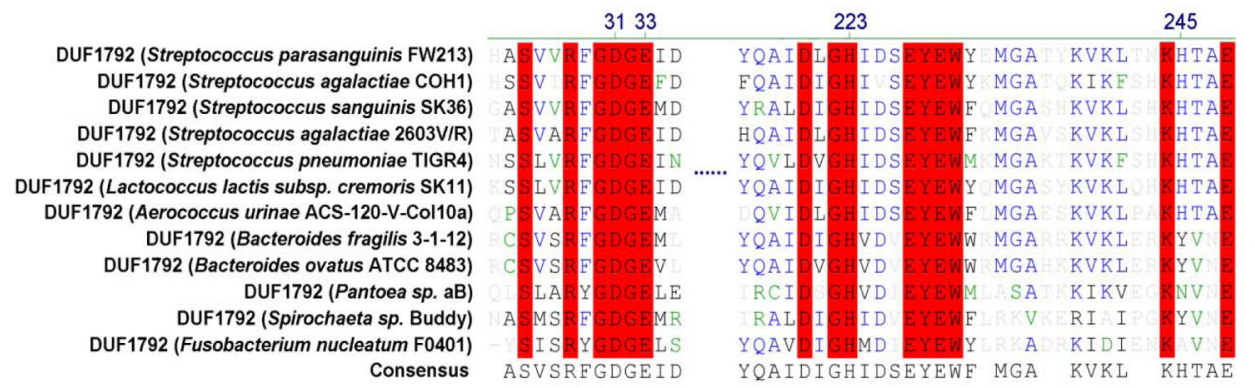

Figure 3. The glycosyltransferase activity and DXE motif of DUF1792 are conserved (a) DUF1792 is functionally conserved. DUF1792 homologs from a variety of bacterial species were cloned into vector pGEx-6p-1 and co-transformed in E. coli carrying rFap1 and Gtf1/2, 3 to determine the ability of DUF1792 to catalyze the transfer of additional sugar residues to Gtf1/2, 3 modified rFap1. Cell lysates from rFap1 (1), Gtf1/2, 3 modified rFap1 (2) and Gtf1/2, 3 modified rFap1coexpressed with dGT1 of S. parasanguinis (3), or with DUF1792 from S. parasanguinis (4), S. agalactiaeCOH1 (5); S. sanguinis SK36 (6), S. agalactiae 2603 V/R (7), S. pneumoniae TIGR4 (8) and F. nucleatum F0401 (9) were subjected to western blotting analysis with Fap1 specific antibody E42. All DUF1792 homologs retarded the migration of rFap1, suggesting they promoted the transfer of additional sugar residues to Gtf1/2, 3 modified Fap1.

(b) Contribution of DUF1792 to glycosylation of Fap1. Wild type dGT1, N-terminal dGT1DUF1792, C-terminal dGT1 constructs were used to complement the dGT1mutant in $S$. parasanguinis. Cell lysates from wild type $S$. parasanguinis (1); Fap1 mutant (2); dGT1 mutant (3); the dGT1 mutant complemented with the dGT1 full-length gene (4), DUF1792 (5); and C-terminal dGT1 (6) were subjected to western blotting analysis with Fap1-peptide specific mAbE42 (top) and anti-DNAK antibody (bottom)as a sample loading control. Complementation of DUF1792 produces an intermediate of Fap1, while the complementation with the C-terminus alone has the same phenotype as dGT1 mutant. (c)The DXE motif and UDP binding sites are highly conserved in bacteria. DUF1792 homologs were identified from a group of streptococci, Lactobacillus lactis and several Gram-negative bacteria. The regions flanking the DGE motif, and UDP binding sites were 
compared. The invariant amino acid residues highlighted in red and consensus sequence was deduced. 
a

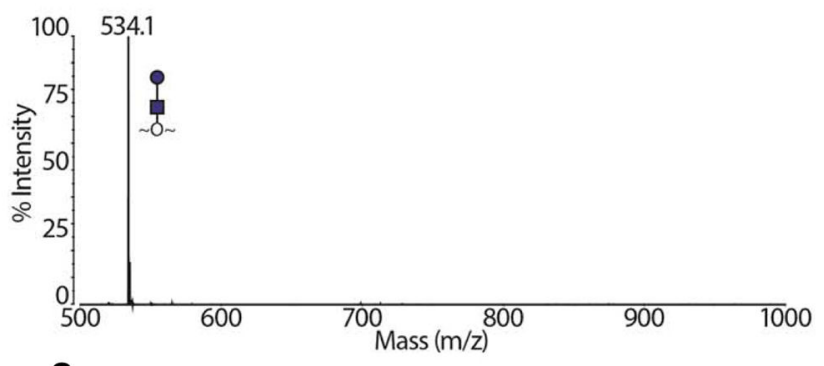

c

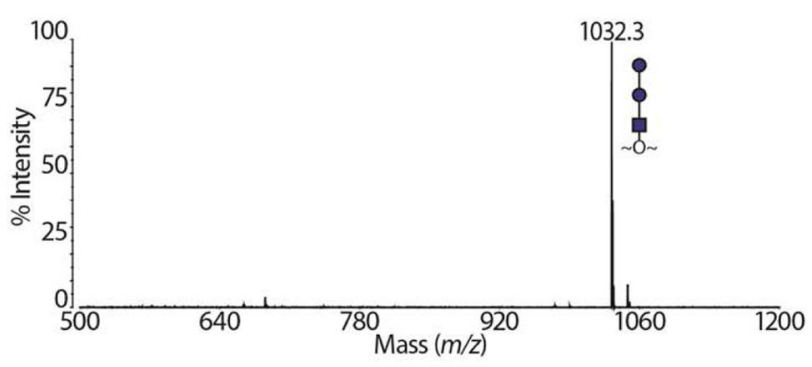

b

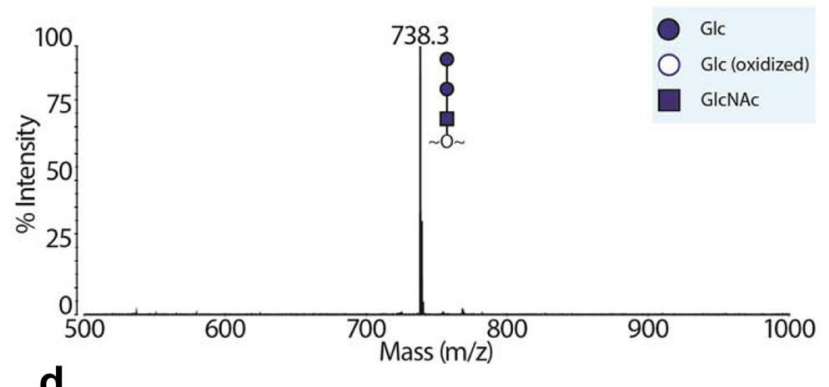

d

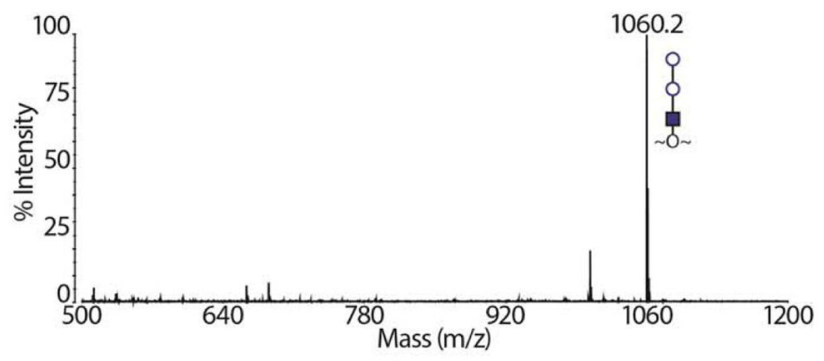

Figure 4. MS spectra confirming in vivo glycosyltransferase activity of DUF1792

The MALDI-TOF spectra of permethylated recombinant Fap1 glycans modified by Gtf1/2/3, and Gtf1/2/3 plus DUF1792 are shown in (a) and (b) respectively. The shift of 204 in $m / z$ corresponding to a permethylated hexose is consistent with the glucosyltransferase activity of DUF1792. Recombinant Fap1 modified by Gtf1/2/3 shown in (b) were peracetylated and oxidized by $\mathrm{CrO}_{3}$. MALDI-TOF spectra of the tri-saccharide from recombinant Fap1 expressed with Gtf1/2/3 and DUF1792, which was analyzed as its peracetylated derivative before and after $\mathrm{CrO}_{3}$ oxidation, are shown in (c) and (d) respectively. A shift of $28 \mathrm{in} \mathrm{m} / \mathrm{z}$ corresponding to two oxidations indicates both linkages are $\beta$. 

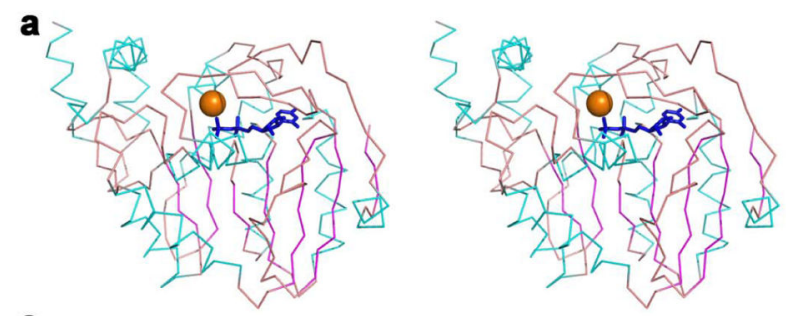

b

C
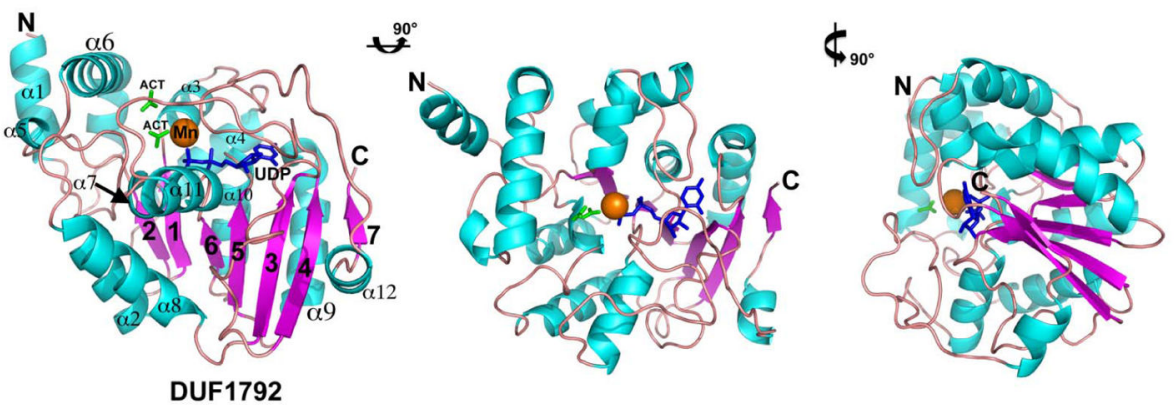

d

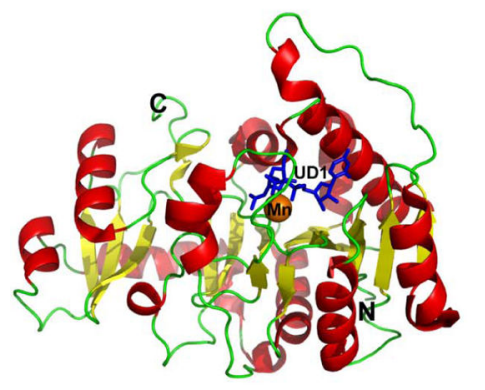

GT-A type (PDB 1FOA, RMSD 4.2A)

e

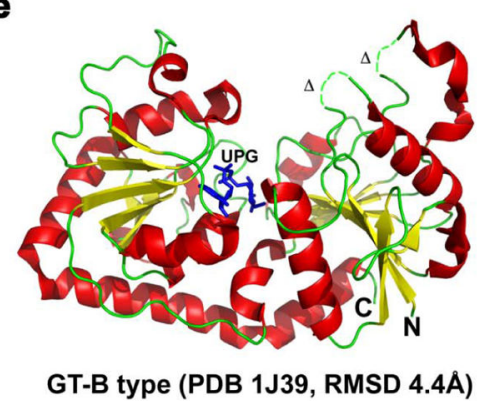

Figure 5. The overall structure of DUF1792 (DUF1972-Mn)

(a) Stereo image of the backbone trace of DUF1792. The alpha helices are colored cyan, the beta-strands are colored magenta, and coiled regions are salmon and the UDP molecule at the active site is shown in blue and the Mn-atom in orange.

(b) Representative 2Fo-Fc electron density for DUF1792-Mn. Electron density for the helical region between residue 223-233 is shown in gray and contoured at 2 sigma.

(c) The overall structure of UDP and Mn-bound DUF1792. The manganese ion is colored orange, the UDP molecule is blue, beta-strands are magenta, alpha-helices are cyan, and coiled regions are salmon. Two acetate ions are found at the active site and are colored green. The secondary structural elements are shown in the figure on the left. Three views are shown (left, middle and right).

(d) Archetypical GT-A type glycosyltransferase(PDB id code 1FOA).

(e) Archetypical GT-B type glycosyltransferase(PDB id code 1J39). 
a

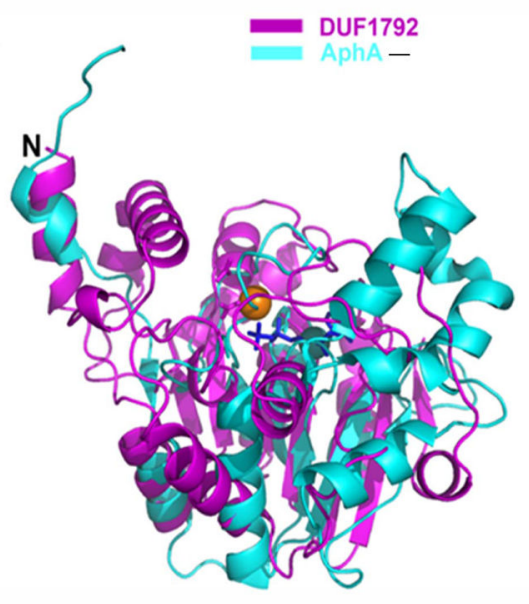

b

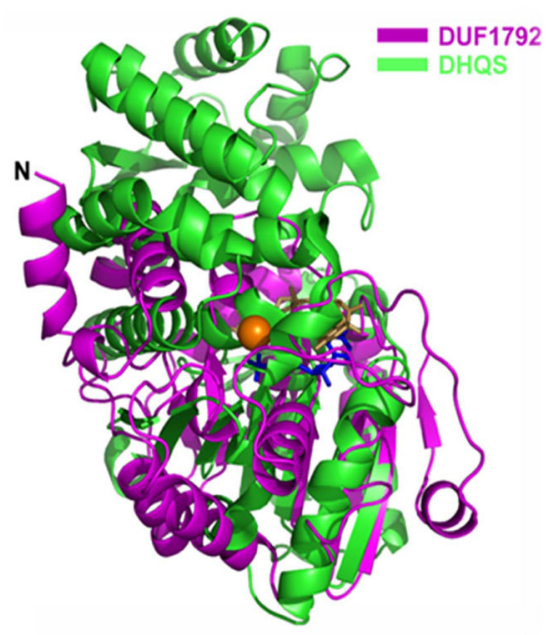

Figure 6. Structural alignment of DUF1792

(a) DUF1792 superimposed onto molecule A of AphA(PDB id code 1Z5G). DUF1792 is shown in magenta cartoon, and AphA is shown in cyan cartoon. The UDP molecule in DUF1792 is shown as stick and colored in blue and the Mn ion shown as an orange sphere. (b) DUF1792 superimposed onto molecule A of 3-Dehydroquinate Synthase (DHQS) (PDB id code 3OKF). DUF1792 is shown in magenta cartoon, and 3-Dehydroquinate Synthase is shown in green cartoon. The UDP molecule in DUF1792 is shown as blue stick and the Mn ion shown as an orange sphere. The nicotinamide-adenine-dinucleotide is shown in sand color stick along with the phosphate ion. 
a

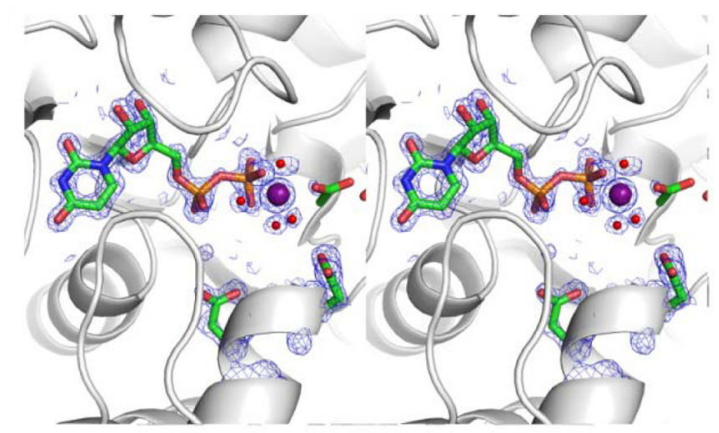

C

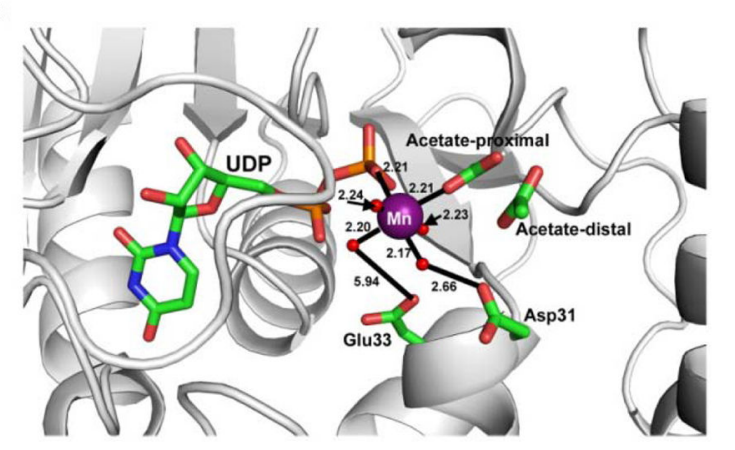

b

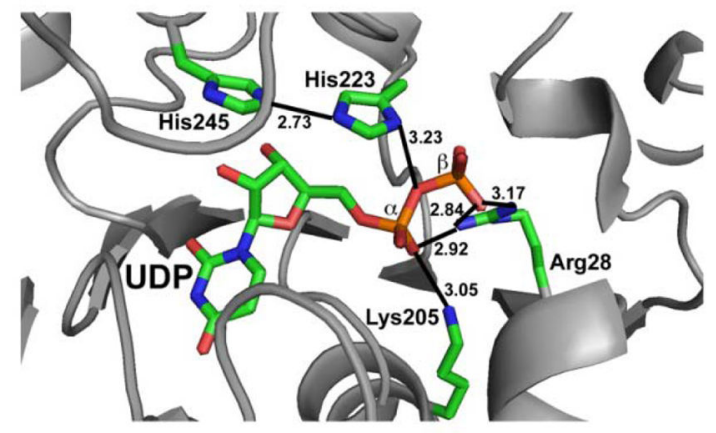

d

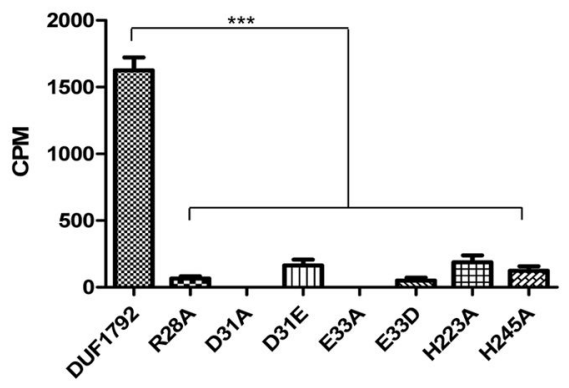

Figure 7. DUF1792 possesses UDP and Manganese binding sites (a) Cross-eyed stereo view showing the electron density of UDP and the manganese ion at the active site. The map shown is a simulated annealing composite omit electron density map calculated in Phenix and contoured at $2.0 \sigma$.

(b) The UDP binding site. UDP and the amino acid residues involved in UDP binding are labeled and atomic distances are shown in Ångströms.

(c) Manganese binding sites. Manganese and amino acid residues involved in manganese binding are shown and labeled. The atomic distances are shown in Ångströms.

(d) Critical residues within UDP and metal binding sites required for glycosyltransferase activity of DUF1792. Site-direct mutagenesis was carried out to mutate critical residues that are involved in binding to UDP and $\mathrm{Mn}^{2+}$, the mutant DUF1792 protein variants were assayed for their in vitro glycosyltransferase activity. The value obtained from three different experiments represent means \pm standard errors of the means. Significant differences were indicated by asterisks $(* * * P<0.001)$. 
a

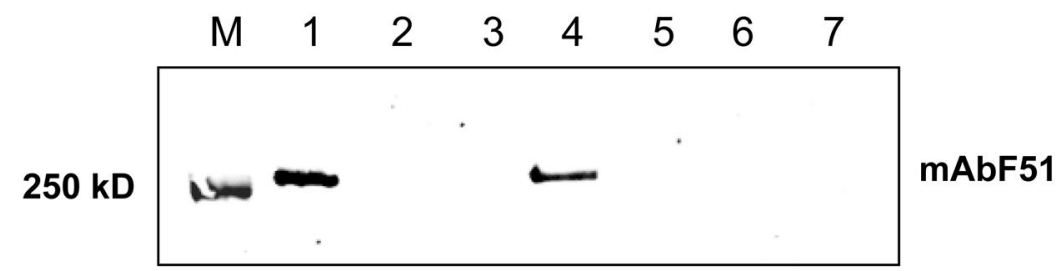

b

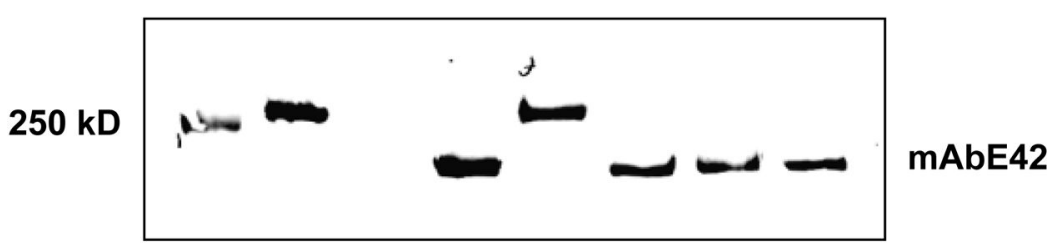

C

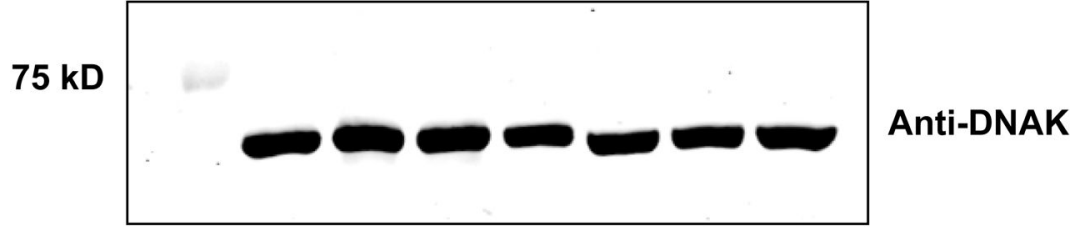

Figure 8. UDP and metal binding are required for Fap1 glycosylation in vivo Wild type and dGT1 site-directed mutant constructs were used to complement the dGTImutant in S. parasanguinis. Cell lysates from S. parasanguinis (1); Fap1 mutant (2); dGT1 mutant (3); the dGT1 mutant complemented with the dGT1 full-length gene (4), dGT1 (D31A) (5); dGT1(D31E) (6); and dGT1(H223A) (7) were subjected to western blotting analysis with Fap1-peptide specific mAbE42 (a), mature Fap1-specific mAbF51 (b) to determine Fap1 production, and anti-DNAK antibody (c) as a sample loading control. 


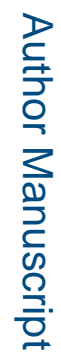

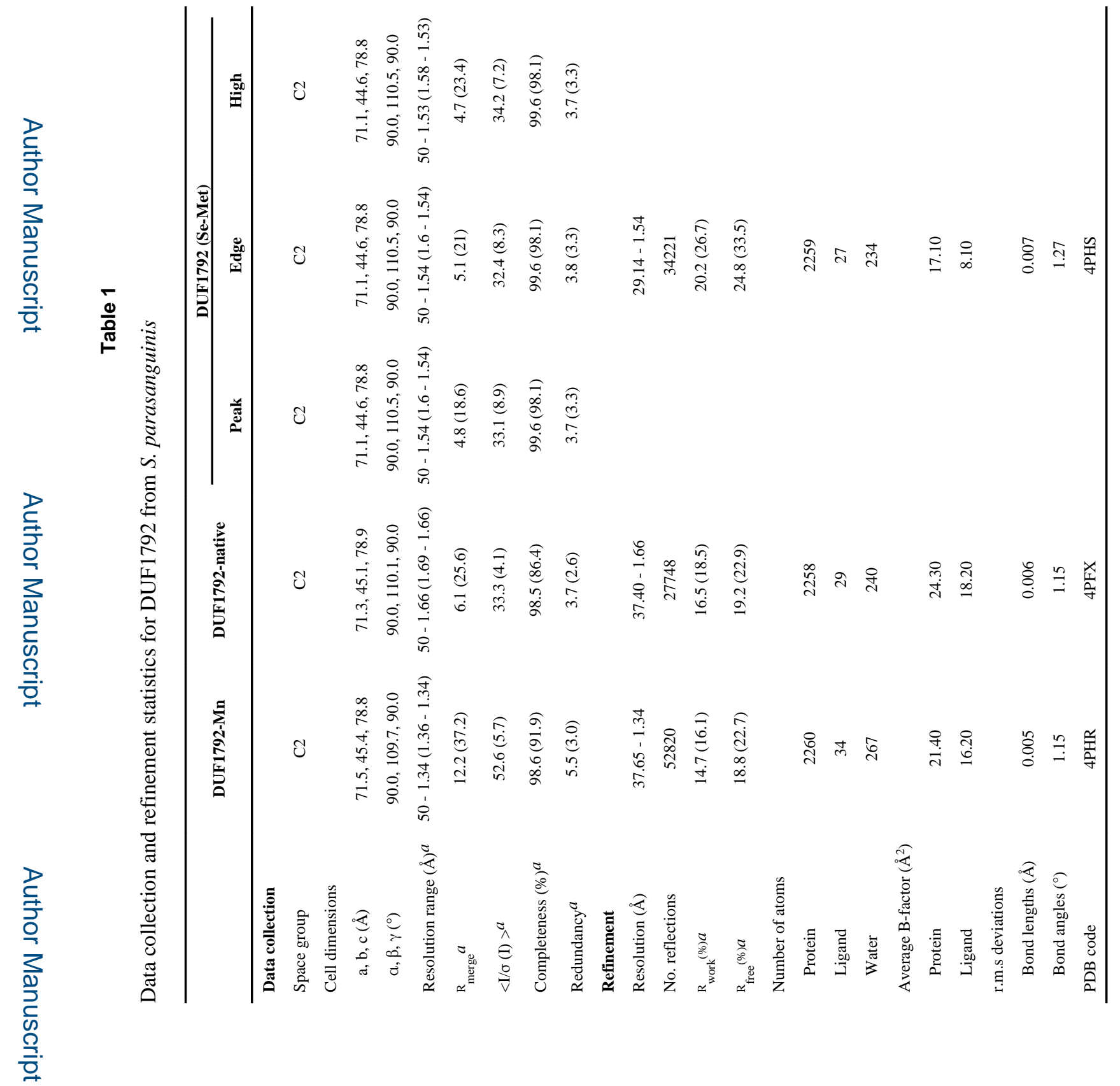

Nat Commun. Author manuscript; available in PMC 2015 March 08. 
